\title{
Stressful life events and the onset of asthma
}

\author{
R. Lietzén*, P. Virtanen*,\#, M. Kivimäki ${ }^{{ }^{\top},+, \S}$, L. Sillanmäki ${ }^{f}$, \\ J. Vahtera ${ }^{\S, * *}$ and M. Koskenvuo
}

ABSTRACT: The status of stressful life events as a risk factor for asthma is unclear and may be dependent on pre-existing allergic rhinitis. This study examined whether exposure to stressful life events predicted the onset of asthma in adults.

This is a prospective, population-based cohort study of 16,881 males and females, aged 20-54 yrs and free of diagnosed asthma at the beginning of the follow-up (January 1, 2004). Data about stressful life events were gathered with a postal survey. The onset of asthma was ascertained through national registers until December 31, 2005.

During the follow-up period, 192 incident cases of asthma were identified. High total exposure to stressful life events, as indicated by a cumulative severity score, predicted the onset of asthma (hazard ratio $1.96,95 \% \mathrm{Cl} 1.22-3.13$ ). This association was robust to adjustment for demographics, smoking and having a cat/dog at home and it was observed both among those with and without allergic rhinitis at baseline. Of the $\mathbf{1 0}$ most stressful life events, the illness of a family member, marital problems, divorce or separation and conflicts with a supervisor were associated with the onset of asthma.

Our study suggests that stressful life events may increase the onset of asthma.

\section{KEYWORDS: Asthma, asthma epidemiology, asthma onset, stressful life events}

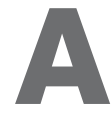
sthma is a disorder of the airways in which chronic inflammation is associated with airway hyperresponsiveness, leading to recurrent episodes of wheezing, breathlessness, chest tightness and coughing [1]. It is one of the most common chronic diseases worldwide, especially in industrialised countries. Among the adult population, the reported prevalence figures vary from $1.9 \%$ in Greece to $18.4 \%$ in Scotland [2]. In Finland, the prevalence of asthma is $4 \%$ among males and $7 \%$ among females [3] and the overall incidence rate during adulthood (i.e. 21-63 yrs) is 0.9 new cases per 1,000 person-yrs [4].

A large number of studies have examined the biological risk factors for asthma. There is strong support for an increased risk of asthma that is associated with genetic factors, allergens, respiratory infections, air pollutants, tobacco smoke [5] and allergic rhinitis (AR) [6]. Recent studies suggest that AR and asthma, in fact, comprise a single syndrome known as the chronic allergic respiratory syndrome, which may affect one or two parts of the respiratory tract [7].

Recently, the role of psychosocial stress as a risk factor for asthma has gained increased attention. Stress is considered to affect the expression of asthma through multidimensional endocrine, neural, immune and behavioural processes [8], but clinical research to date has clarified only part of the causal mechanisms that may link stress to asthma [9]. There is observational evidence that severe negative life events may increase the risk of children's asthma attacks over subsequent weeks [10] and may be associated with asthma symptoms among adolescents [11]. The association between stressful life events and an increased risk of asthma has also been reported in four retrospective studies [12-15] and in at least one prospective study [16] among adults. In the latter study, which was based on 4,010 middle-aged participants in one town and its surroundings, breaking off a life partnership predicted incident asthma, but no other stressful events were found to have an effect [16]. To our knowledge, however, no large-scale, populationbased studies that explore the association between stressful life events and the onset of asthma are available.

In this study, we examine the association between stressful life events and the onset of asthma in a large population sample, taking into account pre-existing AR. We sought to determine whether: 1) the total exposure to stressful life
AFFILIATIONS

*Tampere School of Public Health, University of Tampere, Tampere, ${ }^{+}$Dept of Behavioural Sciences, Helsinki,

${ }^{\S}$ Finnish Institute of Occupational Health, Helsinki, and

** Dept of Public Health, University of Turku and Turku University Hospital, Turku, Finland.

\#Dept of Public Health and Clinical Medicine, University of Umeå, Umeå, Sweden.

-Dept of Epidemiology and Public Health, University College London Medical School, London, UK.

CORRESPONDENCE

R. Lietzén

Dept of Public Health

FIN-20014 University of Turku

Turku

Finland

E-mail: raija.lietzen@utu.fi

Received:

Accepted after revision:

Sept 262010

First published online:

Oct 282010 ${ }^{f}$ Dept of Public Health, University of

Oct 162009 
events is associated with the onset of asthma among adults; 2) specific severe life events are particularly predictive of such an onset; and 3) the association between life events and the asthma onset is dependent on pre-existing AR.

\section{MATERIALS AND METHODS Study population and design}

Data were drawn from the Health and Social Support (HeSSup) study, a longitudinal study on a population sample that is representative of the Finnish population in the following four age groups: 20-24, 30-34, 40-44 and 50-54 yrs at baseline (phase one) [17]. Baseline characteristics were obtained from the phase one postal survey conducted in 1998. Exposure to life events during a 5-yr period between phase one and phase two was assessed with a follow-up questionnaire that was sent to all participants who were still living in Finland in 2003. Of the 25,901 respondents at baseline, 216 died before phase two, 234 had moved abroad and 969 could not be reached due to unknown addresses; therefore, a total of 1,419 baseline respondents did not participate in the follow-up survey. Of all 19,629 respondents at phase two, which revealed an $80 \%$ response rate, 18,900 consented to the use of their recorded health information in Finnish national registers. Out of that pool, we excluded any participants with prevalent asthma or asthma history at phase two $(n=2,016)$. Thus, the final sample consisted of 16,881 participants (6,663 males and 10,218 females) who had no indication of asthma at the beginning of the follow-up (fig. 1). They were followed for a 2-yr period from national health registers in order to detect the onset of new asthma cases (2004-2005) after the phase two survey.

\section{Stressful life events}

Life events that occurred between phases one and two were measured by a list of 21 negative events, as in earlier studies [18-20]. For each event, the questionnaire included the following categories: never, within the previous 6 months, within the previous $5 \mathrm{yrs}$ and $>5$ yrs ago. The subjects classified the severity of each event as: 1, not so burdensome; 2 , burdensome; and 3, extremely burdensome. The events that had occurred during the previous $5 \mathrm{yrs}$ (i.e. in the period

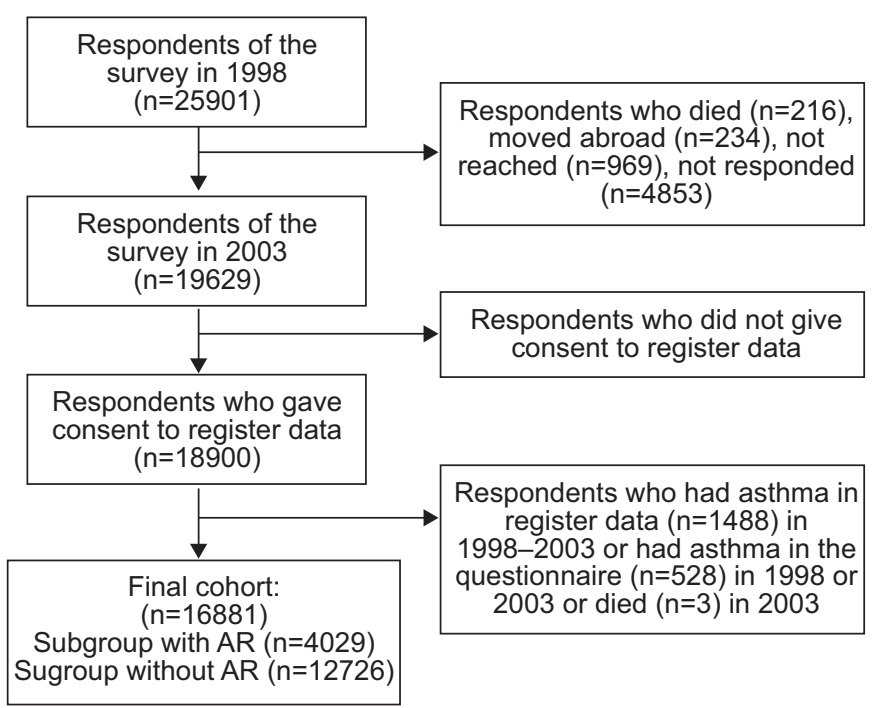

FIGURE 1. Flow chart of the sample selection procedure. AR: allergic rhinitis between the questionnaires) were considered in this study. We excluded 'illness causing work disability of $>21$ days' and 'retirement' because such events might be related to asthma. To assess the total exposure to life events within the 5 yrs of follow-up for each individual, we calculated, from the 2003 survey responses, a cumulative sum score of the severity of events that had occurred within the previous 6 months or 5 yrs. As weights, we used means of squared severity ratings for each event as in our earlier studies [20,21]. Those with a zero score were defined as having no exposure. The exposed were divided into tertiles: light (cumulative sum score 2.74-5.53), moderate (6.15-12.07) and heavy (12.09-55.41). In addition to the total exposure to stressful life events, we measured the nine most burdensome categories of specific events: death of a family member (death of own child, weight (w) 7.42; death of a spouse, w 7.01); emotional, physical or sexual violence ( $w$ 6.16), severe illness in a family member ( $w 5.53$ ), death of the mother ( $w 5.04$ ), a major increase in marital problems (w 4.92), divorce or separation ( w 4.75), severe conflicts with a supervisor ( $w 4.63)$, severe financial difficulties ( $w 4.37$ ) and death of the father ( $w$ 4.25) [20].

\section{Case definition for asthma}

Using the unified personal identification code system, which covers all Finnish citizens, we linked the survey responses to records from three independent and comprehensive Finnish national health registers in order to identify incident cases of asthma. The identification of the cases was based on the clinical diagnosis from two registers (i.e. the Drug Reimbursement Register and the Hospital Discharge Register) of the treating physician (for special reimbursement for medicine costs or hospitalisation) or detailed information about the prescribed medication (the Drug Prescription Register). A participant was classified as having incident asthma when the caseness was verified for the first time by at least one of the three data sources between January 1, 2004 and December 31, 2005.

First, we used the Drug Reimbursement Register of the Social Insurance Institution of Finland, which contains information on persons entitled to special reimbursement for certain chronic diseases, such as asthma. Patients who apply for special reimbursement must attach a detailed medical certificate that was prepared by the treating physician, who also provides data to confirm the diagnosis. The application is then reviewed by a physician in the Social Insurance Institution to determine whether the uniformly defined requirements for the disease are met. From this register, participants were defined as incident asthma cases if they were recorded in the Central Drug Register as being eligible for asthma treatment for the first time during the follow-up.

Secondly, we used prescription data to assess the beginnings of medical treatment for asthma. In Finland, the National Social Insurance Scheme at the Social Insurance Institution provides basic reimbursement (currently $42 \%$ ) for all filled outpatient prescriptions that are recorded in the Drug Prescription Register according to the World Health Organization's Anatomical Therapeutic Chemical (ATC) Classification [22] and by the date of purchase. We identified all participants with two or more prescriptions for drugs for obstructive airway diseases (ATC code R03) in any year during the follow-up by using the day of the first purchase as an indicator of the onset of asthma. 
Thirdly, we obtained data from the Hospital Discharge Register of the National Institute for Health and Welfare, which includes records on all in-patient hospital admissions. This register is comprised of countrywide information on virtually all hospitalisations. We obtained the discharge dates and the corresponding main diagnoses for hospitalisation due to asthma (International Classification of Disease-10 J45).

All of the individuals who were identified as having an onset of asthma in any of these registers before January 1, 2004 were excluded from the analysis, as were those individuals who reported a life-time diagnosis of asthma in either survey. Of all 192 incident cases of asthma that were detected on any of the three registers, 190 (99\%) cases had purchases of prescribed medication for asthma and $41(21 \%)$ cases had a clinical diagnosis of asthma noted in hospitalisation or special reimbursement records. Of the latter, 39 (95\%) cases also had purchases of prescribed medication for asthma.

\section{Baseline characteristics}

All baseline characteristics were measured at phase one. The possible risk factors for the development of asthma that were considered in this study included AR, exposure to dogs or cats [5] and smoking [23]. The participants were asked whether a doctor had previously told them that they have or have had AR (yes/no). The subjects were classified as having AR versus not having AR (baseline). AR is a risk factor for asthma, particularly for the allergic phenotype [6, 24]. Information about a pet dog/cat was obtained by asking whether the participant has a pet. The variable was dichotomised as having a pet dog/cat at home versus not having one. The participants reported their current and past smoking habits, smoking rate and number of cigarettes [17]. They were classified as nonsmokers, past smokers or current smokers. Sociodemographic background variables included sex, age group, level of education (basic/vocational/college/university) and marital status (single, divorced or widowed versus married or cohabiting).

\section{Statistical analysis}

All analyses were performed by using PASW Statistics for Windows software, Release 18.0.0 (SPSS Inc., Chicago, IL, USA). The associations between the baseline characteristics and the onset of asthma, as well as those between life events that occurred between phase one and phase two and the onset of asthma, were analysed via the Cox proportional hazard model. Follow-up began on January 1, 2004 (i.e. the year after the phase two survey) and ended upon the first occurrence of the outcome measure, death, or December 31, 2005, whichever came first. We calculated hazard ratios and their $95 \%$ confidence intervals.

We studied the associations of baseline characteristics and risk factors with the onset of asthma during the risk time. Then we studied the association between the total exposure to life events and onset of asthma, first adjusting the analysis for all demographics and finally adding the risk factors to the model. Adjusting in the same way, we analysed the association between the 10 most stressful life events and asthma onset in the risk time.

The sex difference in the association of the cumulative severity score to the onset of asthma was assessed with the interaction term gender* cumulative severity score. Because no significant interaction was found $(p=0.43)$ we analysed males and females in combination adjusting for sex.

We performed two sensitivity analyses. First, we tested whether the effect of life events on asthma were dependent on AR. This analysis was carried out among two subgroups: participants with $(n=4,029)$ and without AR $(n=12,726)$. Secondly, we tested whether the association between life events and the subsequent onset of asthma was dependent on case definition. This analysis was carried out in subgroups of participants whose case definition was based on the clinical diagnosis of the treating physician (i.e. special reimbursement for medicine costs or hospitalisation, $n=41$ ) and those whose caseness was determined according to prescribed medication $(n=190)$.

\section{RESULTS}

During a mean \pm SD follow-up of $2.0 \pm 0.12$ yrs, 192 participants were diagnosed with asthma. Past smoking habits, older age and the presence of AR were associated with an increased risk (table 1).

\begin{tabular}{|c|c|c|c|}
\hline TABLE 1 & \multicolumn{3}{|c|}{$\begin{array}{l}\text { Characteristics of study participants and hazard } \\
\text { ratios (HRs) for incident asthma (all variables in } \\
\text { the same model) }\end{array}$} \\
\hline Characteristic & Participants & Cases & HR $(95 \% \mathrm{CI})$ \\
\hline \multicolumn{4}{|l|}{ Sex } \\
\hline Males & $6663(40)$ & $61(0.9)$ & 1.00 \\
\hline Females & $10218(60)$ & $131(1.3)$ & $1.33(0.96-1.85)$ \\
\hline \multicolumn{4}{|l|}{ Age group } \\
\hline 20-24 yrs & $4268(25)$ & $39(0.9)$ & 1.00 \\
\hline $30-34$ yrs & $3814(23)$ & $42(1.1)$ & $1.38(0.85-2.24)$ \\
\hline 40-44 yrs & $4200(25)$ & $52(1.2)$ & $1.43(0.89-2.29)$ \\
\hline $50-54$ yrs & 4599 (27) & $58(1.3)$ & $1.60(1.01-2.51)$ \\
\hline \multicolumn{4}{|l|}{ Marital status } \\
\hline Single & $5303(31)$ & $61(1.2)$ & 1.00 \\
\hline Married & $11560(69)$ & $130(1.1)$ & $0.90(0.64-1.27)$ \\
\hline \multicolumn{4}{|c|}{ Level of education } \\
\hline University & $2824(17)$ & $28(1.0)$ & 1.00 \\
\hline College & $4954(30)$ & $55(1.1)$ & $1.12(0.68-1.81)$ \\
\hline Vocational & 4024 (24) & $38(0.9)$ & $0.95(0.56-1.62)$ \\
\hline Basic & 4902 (29) & $65(1.3)$ & $1.39(0.86-2.27)$ \\
\hline \multicolumn{4}{|l|}{ Smoking } \\
\hline Nonsmoker & $7298(47)$ & $64(0.9)$ & 1.00 \\
\hline Past smoker & $4301(28)$ & $67(1.6)$ & $1.66(1.16-2.37)$ \\
\hline Current smoker & 3904 (25) & $50(1.3)$ & $1.35(0.93-2.02)$ \\
\hline \multicolumn{4}{|l|}{ Dog/cat at home } \\
\hline No & $10556(63)$ & $111(1.1)$ & 1.00 \\
\hline Yes & $6189(37)$ & $77(1.2)$ & $1.23(0.91-1.99)$ \\
\hline \multicolumn{4}{|l|}{ Allergic rhinitis } \\
\hline No & 12726 & $112(0.9)$ & 1.00 \\
\hline Yes & 4029 & $76(1.9)$ & $2.21(1.62-3.01)$ \\
\hline
\end{tabular}

Data are presented as $n(\%)$, unless otherwise stated. The total number of study participants was 16,881 . 
TABLE 2 Exposure to stressful life events (cumulative severity score) as a predictor of incident asthma

\begin{tabular}{|c|c|c|c|c|}
\hline Life event severity score & Subjects $\mathrm{n}^{\#}$ & Model $1^{\circ}$ & Model 2 & Model $3^{\circ}$ \\
\hline \multicolumn{5}{|l|}{ All participants } \\
\hline$>0-5.53$ & 4136 & $1.35(0.83-2.19)$ & $1.35(0.83-2.20)$ & $1.35(0.83-2.19)$ \\
\hline $6.15-12.07$ & 3835 & $1.20(0.72-1.99)$ & $1.20(0.73-2.00)$ & $1.19(0.72-1.98)$ \\
\hline$>12.09$ & 3837 & $1.96(1.23-3.12)$ & $2.01(1.26-3.20)$ & $1.96(1.22-3.13)$ \\
\hline$>0-5.53$ & 3154 & $1.42(0.77-2.60)$ & $1.42(0.77-2.61)$ & $1.41(0.77-2.60)$ \\
\hline $6.15-12.07$ & 2911 & $1.02(0.53-1.97)$ & $1.03(0.53-1.99)$ & $1.02(0.53-1.97)$ \\
\hline$>12.09$ & 2772 & $1.83(1.01-3.31)$ & $1.88(1.04-3.43)$ & $1.84(1.01-3.35)$ \\
\hline \multicolumn{5}{|l|}{ With allergic rhinitis } \\
\hline 0 & 622 & 1.00 & 1.00 & 1.00 \\
\hline$>0-5.53$ & 955 & $1.08(0.47-2.47)$ & $1.13(0.49-2.58)$ & $1.13(0.49-2.59)$ \\
\hline
\end{tabular}

\section{Stressful life events and asthma}

Altogether, 13,050 (79\%) participants in our study reported that they encountered a stressful event during the 5-yr exposure window between phase one and phase two. Of these participants, 4,659 (36\%) experienced one event, 7,846 (47\%) experienced two to five events and 545 (3\%) endured six to 12 events. There were 3,366 participants who reported no events.

The cumulative severity rating of the events ranged from 0 to 55.41 (median 6.97). Table 2 shows that those in the highest tertile of exposure to life events had a two times (95\% CI 1.26-3.20) greater risk of asthma during the follow-up, compared with participants who did not experience any stressful life events. This relative risk remained unchanged after taking into account adjustments for demographics, smoking habits and exposure to dogs or cats.

Analyses of specific life events showed that seven of the nine categories of the most severe events were associated with a 1.5-1.7-fold risk of asthma during the follow-up period (table 3). Statistically, a significantly increased risk of asthma onset was observed after a divorce or separation, major increase in marital problems, severe illness in a family member, severe conflicts with a supervisor and severe financial difficulties. These associations were robust to all adjustments with one exception; the category 'severe financial difficulties' was not associated with asthma risk in the fully adjusted model. The only categories that showed no association

TABLE 3 Most severe specific life events as predictors of incident asthma

\begin{tabular}{|c|c|c|c|c|c|}
\hline Life event during 5 yrs & $\begin{array}{c}\text { Subjects in the } \\
\text { analysis }\end{array}$ & $\begin{array}{c}\text { Subjects with the } \\
\text { event }\end{array}$ & Model $1^{\circ}$ & Model $2^{\circ}$ & Model $3^{*}$ \\
\hline Death of a family member ${ }^{+}$ & 203 & 155 & $0.85(0.21-3.42)$ & $0.71(0.17-2.88)$ & $0.72(0.18-2.93)$ \\
\hline Violence & 792 & 149 & $1.48(0.84-2.61)$ & $1.44(0.81-2.56)$ & $1.39(0.78-2.47)$ \\
\hline Severe illness in a family member & 2652 & 133 & $1.52(1.06-2.19)$ & $1.47(1.01-2.12)$ & $1.47(1.01-2.12)$ \\
\hline Death of mother & 1106 & 134 & $1.57(0.97-2.56)$ & $1.41(0.83-2.41)$ & $1.40(0.82-2.39)$ \\
\hline Major increase in marital problems & 2773 & 135 & $1.62(1.12-2.33)$ & $1.47(1.01-2.12)$ & $1.47(1.01-2.12)$ \\
\hline Divorce or separation & 1822 & 115 & $1.65(1.08-2.55)$ & $1.75(1.13-2.73)$ & $1.67(1.07-2.62)$ \\
\hline Severe conflicts with supervisor & 1381 & 152 & $1.61(1.04-2.49)$ & $1.65(1.07-2.56)$ & $1.63(1.05-2.52)$ \\
\hline Severe financial difficulties & 2500 & 142 & $1.47(1.01-2.13)$ & $1.41(0.97-2.06)$ & $1.40(0.95-2.05)$ \\
\hline Death of father & 1387 & 100 & $0.85(0.48-1.53)$ & $0.76(0.41-1.43)$ & $0.77(0.41-1.43)$ \\
\hline
\end{tabular}

Data are presented as $\mathrm{n}$ or hazard ratios (HRs) with 95\% confidence intervals. Statistically significant HRs are shown in bold. Model 1: crude; model 2: adjusted for sociodemographic factors (age, sex, marital status and education); model 3: model 2+asthma risk factors (smoking and cat/dog at home). \# : calculated from model 3;

$\because$ : Cox regression models were used; ${ }^{+}$: death of own child or death of spouse. 
with asthma onset were 'death of a family member' and 'death of own father.'

Figure 2 illustrates the cumulative hazard curves of asthma onset by exposure to stressful life events. The expected dispersion of hazard curves between categories of cumulative severity scores was observed across the entire follow-up period ( $\mathrm{p}$ for log rank test $=0.006$ ).

\section{Sensitivity analyses}

To test the robustness of our findings we excluded the participants with AR. This analysis replicated the findings for all participants. The hazard of asthma onset for those who were most severely exposed to life events was 1.9 times higher (95\% CI 1.04-3.43) than that for the non-exposed (table 3). In an analysis that included the participants with AR only, the risk estimate was practically the same. However, it failed to reach statistical significance due to lower numbers. The nonsignificant interaction $(p=0.46)$ between $A R$ and life events is consistent with these findings.

A further sensitivity analysis testing whether the association between life events and a subsequent onset of asthma was dependent on case definition also replicated findings from the main analysis. Determining incident asthma on the basis of drug prescriptions (in analysis $n=187$ ), the hazard ratio for high exposure was 2.09 (95\% CI 1.33-3.33). If incident asthma was determined solely on the basis of clinical diagnosis (i.e. special reimbursement for asthma or hospitalisation, $n=41$ ), the hazard ratio after a high level of exposure to stressful life events remained practically the same but did not reach statistical significance (HR $2.41,95 \%$ CI 0.88-6.63). These associations were adjusted for age and sex; light and moderate exposure scores were combined due to the small number of cases (data not shown).

\section{DISCUSSION}

In this population-based prospective cohort study of almost 17,000 participants who had no indication of asthma at the beginning of the follow-up period, a high exposure to stressful life events was associated with a two-fold increased risk of the subsequent onset of asthma as measured by reliable national

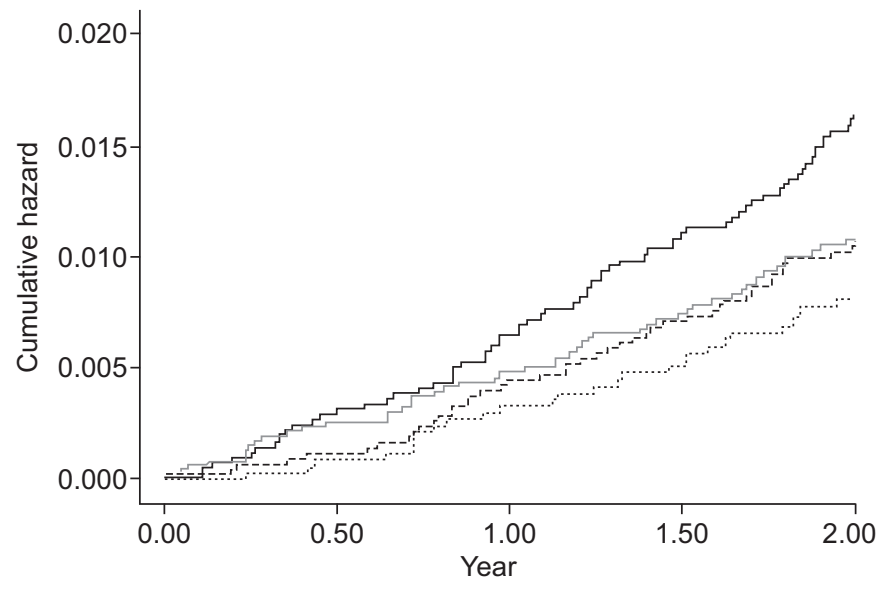

FIGURE 2. Cumulative hazard of asthma onset by exposure to stressful life events. Severity scores of life events: solid black line: high; grey line: low; dashed line: middle; and dotted line: zero. health registers. The elevated risk was not accounted for by baseline characteristics, such as demographics, smoking habits or exposure to dogs or cats and it was of the same magnitude both among those with and without AR. We also found that specific severe events (i.e. a divorce or separation, a major increase in marital problems, severe illness in a family member and severe conflicts with a supervisor) were predictive of the onset of asthma.

Our findings support the biopsychosocial model of stress [25], which suggests that stressful life events may alter the psychological, immunological and endocrine systems and contribute to the onset of asthma. To the best of our knowledge, the relationship between total exposure to negative life events and the onset of asthma has not been previously reported. The findings of this study are in agreement with prior studies that suggest an association of stressful life events with various health problems ranging from sleep disturbances and sickness absence to depression and breast cancer $[19,20,26,27]$.

To date, only a few studies have prospectively investigated the association between stressful life events and adult asthma. The only longitudinal cohort study of which we are aware followed a population-based sample of 4,010 middle-aged adults from Germany via questionnaire surveys at baseline (1992-1995) and follow-up (2002 and 2003) [16]. Exposure to three stressful life events was measured (i.e. unemployment, having broken off a life partnership and death of someone close). Having broken off a life partnership was associated with a risk of incident asthma that is 2.2 times higher, while no excess risk was found for the other two life events. However, this study was based on a self-report of asthma. In addition, some earlier clinical and retrospective studies noted that adults with acute asthma report that they have experienced a divorce or marital problems before the onset of the disease more often than the nonasthmatic controls $[12,14]$. In our study, based on a follow-up of a population sample with no indication of asthma at baseline, individuals who had interpersonal conflicts indicated by divorce or separation, a major increase in marital problems and severe conflicts with a supervisor experienced a risk of incident asthma that was 1.6-1.7 times higher in the future. In agreement with previous studies, the death of someone close, such as a family member or one's own mother or father, was not associated with increased risk of asthma [15, 16]. The fact that the case definition was based on objective indicators of asthma increased the credibility of our findings.

We also noted a robust association between the severe disease of a family member and incident asthma. In line with this result, SALMINEN [14] observed that a severe disease of a family member was more common among acute asthma patients than among non-asthmatic controls. A smaller-scale analysis found, in contrast, no association between family death or illness and diagnosed asthma [15]. It is noteworthy that there are some differences between the studies in life event variables in the analyses and in the study populations. We are not aware of any other studies on violence or financial difficulties as predictors of the onset of asthma and, in this study, no robust association between these events and asthma onset was found.

The strengths of this study are that data were included from a large sample of working-age adults, a study design that allowed 
the determination of temporary order between exposure to stressful life events and the onset of the disease and the measurement of asthma from national health registers rather than relying on self-reporting. In Finland, the validity of the national registers has been found to be high [28], reasonably accurate and highly reliable for epidemiological study purposes [29]. We were also able to control a number of sociodemographic elements and asthma risk factors. At phase one, the response rate was relatively low $(40 \%)$, and there may have been differences between respondents and non-respondents regarding the frequency of asthma and stressful life events. However, $>80 \%$ of these individuals participated in the phase two survey and, of them, $96 \%$ consented to the linking of data from national health registers. Thus, it is unlikely that the longitudinal associations between life events and incident asthma would be spurious due to the low participation at phase one.

\section{Conclusions}

This is apparently the first epidemiological study of a workingage population that investigates the association between stressful life events and the onset of asthma from reliable health registers. We found that the severity of all stressful life events was associated with the onset of asthma and observed associations with a divorce or separation, a major increase in marital problems, a severe illness in a family member and severe conflicts with a supervisor. Taking into account the nature of asthma as a multifactorial disease, the finding of this study that some life events may trigger the onset of asthma in adults deepens the understanding of the disease's psychosocial risk factors.

\section{SUPPORT STATEMENT}

This study was supported by the Academy of Finland (grant numbers 110451, 117604, 124271, 128089 and 129262). M. Kivimäki and J. Vahtera were supported by the Academy of Finland (projects 124271, 124322, 128089 and 129262). M. Kivimäki is also supported by the BUPA Foundation, UK, the National Institute on Aging (R01AG034454), the National Heart, Lung, and Blood Institute (R01HL036310), NIH, USA and the New OSH ERA Programme grant, EU.

\section{STATEMENT OF INTEREST}

None declared.

\section{REFERENCES}

1 Bateman ED, Hurd SS, Barnes PJ, et al. Global strategy for asthma management and prevention: GINA executive summary. Eur Respir J 2008; 31: 143-178.

2 Masoli M, Fabian D, Holt S, et al. The global burden of asthma: executive summary of the GINA Dissemination Committee report. Allergy 2004; 59: 469-478.

3 Jousilahti $\mathrm{P}$, Heliövaara $\mathrm{M}$, et al. Respiratory and skin diseases. In: Aromaa A, Koskinen S, eds. Health and Functional Capacity in Finland; Baseline Results of the Health 2000 Health Examination Survey. Helsinki, Publications of the National Public Health Institute, 2004; pp. 52-54.

4 Jaakkola MS, Ieromnimon A, Jaakkola JJK. Are atopy and specific IgE to mites and molds important for adult asthma? J Allergy Clin Immunol 2006; 117: 642-648.

5 Gilmour MI, Jaakkola MS, London SJ, et al. How exposure to environmental tobacco smoke, outdoor air pollutants, and increased pollen burdens influences the incidence of asthma. Environ Health Perspect 2006; 114: 627-633.
6 Shaaban R, Zureik M, Soussan D, et al. Rhinitis and onset of asthma: a longitudinal population-based study. Lancet 2008; 372: 1049-1057.

7 Togias A. Rhinitis and asthma: evidence for respiratory system integration. J Allergy Clin Immunol 2003; 111: 1171-1183.

8 Wright RJ, Cohen RT, Cohen S. The impact of stress on the development and expression of atopy. Curr Opin Allergy Clin Immunol 2005; 5: 23-29.

9 Rietveld S, Everaerd W, Creer TL. Stress-induced asthma: a review of research and potential mechanisms. Clin Exp Allergy 2000; 30: 1058-1066.

10 Sandberg S, Paton J, Ahola S, et al. The role of acute and chronic stress in asthma attacks in children. Lancet 2000; 356: 982.

11 Turyk ME, Hernandez E, Wright RJ, et al. Stressful life events and asthma in adolescents. Pediatr Allergy Immunol 2008; 19: 255-263.

12 Teiramaa E.: Psychosocial factors in the onset and course of asthma: a clinical study on 100 patients. PhD thesis. Acta Universitatis Oulu, Series D, Medica No. 14, Psychiatrica No. 4. Oulu, Finland, 1977.

13 Levitan H. Onset of asthma during intense mourning. Psychosomatics 1985; 26: 939-941.

14 Salminen JK. Psychosocial factors in asthma: a study of 50 acute and 31 chronic adult patients. PhD thesis. Publications of the Social Insurance Institution, Turku, Finland, 1985.

15 Kilpeläinen M, Koskenvuo M, Helenius H, et al. Stressful life events promote the manifestation of asthma and atopic diseases. Clin Exp Allergy 2002; 32: 256-263.

16 Loerbroks A, Apfelbacher CJ, Thayer JF, et al. Neuroticism, extraversion, stressful life events and asthma: a cohort study of middle-aged adults. Allergy 2009; 64: 1444-1450.

17 Korkeila K, Suominen S, Ahvenainen J, et al. Non-response and related factors in a nation-wide health survey. Eur J Epidemiol 2001; 17: 991-999.

18 Holmes TH, Rahe RH. The Social Readjustment Rating Scale. J Psychosom Res 1967; 11: 213-218.

19 Lillberg K, Verkasalo PK, Kaprio J, et al. Stressful life events and risk of breast cancer in 10,808 females: a cohort study. Am J Epidemiol 2003; 157: 415-423.

20 Vahtera J, Kivimaki M, Hublin C, et al. Liability to anxiety and severe life events as predictors of new-onset sleep disturbances. Sleep 2007; 30: 1537-1546.

21 Korkeila J, Vahtera J, Nabi H, et al. Childhood adversities, adulthood life events and depression. J Affect Disord 2010; 127: 130-138.

22 World Health Organization. Guidelines for ATC classification and DDD assignment. 7th Edn. Oslo, WHO Collaborating Centre for Drug Statistics Methodology, 2004.

23 Piipari R, Jaakkola JJK, Jaakkola N, et al. Smoking and asthma in adults. Eur Respir J 2004; 24: 734-739.

24 Torén K, Hermansson BA. Incidence rate of adult-onset asthma in relation to age, sex, atopy and smoking: a Swedish populationbased study of 15813 adults. Int J Tub Lung Dis 1999; 3: 192-197.

25 Wright RJ, Rodriguez M, Cohen S. Review of psychosocial stress and asthma: an integrated biopsychosocial approach. Thorax 1998; 53: 1066-1074.

26 Vahtera J, Pentti J, Helenius H, et al. Sleep disturbances as a predictor of long-term increase in sickness absence among employees after family death or illness. Sleep 2006; 29: 673-682.

27 Kivimäki M, Vahtera J, Elovainio M, et al. Death or illness of a family member, violence, interpersonal conflict, and financial difficulties as predictors of sickness absence: longitudinal cohort study on psychological and behavioural links. Psychosom Med 2002; 64: 817-825.

28 Gissler M, Haukka J. Finnish health and social welfare registers in epidemiological research. Norsk Epidemiologi 2004; 14: 113-120.

29 Rapola JM, Virtamo J, Korhonen P, et al. Validity of diagnoses of major coronary events in national registers of hospital diagnoses and deaths in Finland. Eur J Epidemiol 1997; 13: 133-138. 